\section{Consumo de cafeína e de alimentos-fonte de cafeína e prematuridade: um estudo caso-controle}

\author{
Caffeine intake and food sources of caffeine \\ and prematurity: a case-control study
}

1 Instituto de Medicina Social, Universidade do Estado do Rio de Janeiro, Rio de Janeiro, Brasil.

Correspondência R. A. G. Souza Departamento de Epidemiologia, Instituto de Medicina Social, Universidade do Estado do Rio de Janeiro. Rua General Clarindo 488 apto. 103, bloco 2 Rio de Janeiro, $R J$ 20755-320, Brasil. ritadriana@ims.uerj.br ritadriana@ibest.com.br

\section{Abstract}

Caffeine (1,3,7-trimethylxanthine) is an alkali that easily crosses the placental barrier and can interfere in the growth and development of fetal cells and compromise fetal oxygenation. Considering the widespread consumption of foods containing caffeine in Brazil, the aim of this study was to evaluate the association between total caffeine consumption (including its food sources) and prematurity. A case-control study of 140 cases (newborns with gestational age less than 37 weeks) and 162 controls (newborns with 37 weeks gestational age or greater) evaluated caffeine consumption during pregnancy. Intake measurement used a semi-quantitative food frequency questionnaire based on the following foods: coffee, tea, and powdered chocolate. Total caffeine consumption (including food sources) during pregnancy was not associated with prematurity, and most intakes were less than 300mg/ day. Caffeine consumption in the present study does not support guidelines against caffeine consumption by Brazilian pregnant women.

Caffeine; Pregnancy; Premature Labor
Rita Adriana Gomes de Souza ${ }^{1}$ Rosely Sichieri 1

\section{Introdução}

O interesse pelo estudo da cafeína começou em meados da década de 70, quando estudos em animais indicaram que a cafeína estaria relacionada a uma diminuição no crescimento, redução do peso ao nascer e anormalidades esqueléticas 1,2 .

Em 1980, com base nos achados de estudos em animais, o Food and Drug Administration dos Estados Unidos (FDA) sugeriu que as mulheres grávidas evitassem ou diminuíssem o consumo de alimentos e/ou bebidas contendo cafeína ${ }^{3}$.

A cafeína (1,3,7-trimetilxantina) é uma metilxantina que facilmente atravessa a barreira placentária, com quantidades substanciais passando para o líquido amniótico, sangue do cordão umbilical, plasma e urina dos neonatos 4 . Suas maiores fontes alimentares são café, chá, chocolate e refrigerantes do tipo cola 5 . Cerca de 2 mil drogas também contêm cafeína e 25 dessas podem ser usadas na gravidez 6 . O seu consumo é tão amplo que $98,0 \%$ das mulheres em idade reprodutiva consomem regularmente cafeína, seja através da alimentação ou através de medicação, sendo que $72,0 \%$ dessas mulheres continuam usando cafeína durante a gravidez 7 .

A depuração da cafeína fica alterada durante a gravidez, sobretudo no segundo e terceiro trimestres, quando a meia-vida da cafeína é de cerca de 7 e 10 horas, respectivamente, enquanto que em mulheres não-grávidas é de 2,5 a 4,5 
horas 8,9. Este aumento da meia-vida da cafeína nas mães coincide com o período durante $o$ qual o desenvolvimento fetal é exponencial e uma acumulação desta substância poderia representar um potencial risco para o feto 10 .

O aumento da concentração de cafeína inibe a fosfodiesterase e esta enzima degrada a adenosina monofosfato cíclica (cAMP), elevando, desta maneira, os níveis de cAMP, aumento este que pode interferir no crescimento e desenvolvimento das células fetais 11,12. Ainda, pelo efeito vasoconstrictor da cafeína pelo aumento das catecolaminas (especialmente a epinefrina), o suplemento fetal de oxigênio poderia ficar comprometido 13. Além disso, dada a similaridade química entre a cafeína e componentes do ácido desoxiribonucléico (DNA), investigadores têm se interessado pelo potencial mutagênico da cafeína 14,15,16. A possível incorporação de cafeína no material genético pode alterar as instruções de replicação celular 15,17, diminuindo a fase G2 da mitose e, conseqüentemente, o tempo para reparo do dano no cromossomo, aumentando, dessa maneira, o percentual de células mortas 18. Embora a cafeína seja mutagênica em organismos inferiores e sistemas celulares simples 19 , seu efeito mutagênico em humanos é ainda incerto 20.

Os estudos epidemiológicos sobre os efeitos da cafeína no desenvolvimento do feto parecem indicar associação somente com consumo elevado 21,22,23. Em 1998, foram publicadas duas meta-análises sobre o consumo de cafeína e desenlaces na gravidez. A primeira 4 encontrou uma odds ratio de Mantel-Haenszel $\left(\mathrm{OR}_{\mathrm{MH}}\right)$ de 1,36 (intervalo de confiança de 95,0\% - IC95\%: 1,29-1,45) para aborto espontâneo em 42.988 gestações e $\mathrm{OR}_{\mathrm{MH}}$ de 1,51 (IC95\%: 1,39-1,63) para baixo peso ao nascer em 64.268 gestações, para consumo maior que $150 \mathrm{mg}$ de cafeína/dia. Já a segunda 24 sugeriu um provável efeito da cafeína no peso ao nascer, mas não para prematuridade. Como os estudos dessa segunda meta-análise foram muito heterogêneos, não houve a possibilidade de se obter uma medida sumária. Dado o amplo consumo de alimentos contendo cafeína no Brasil, justificase avaliar a possibilidade dessa associação, sendo o objetivo do presente trabalho estudar a associação entre o consumo de cafeína total e dos alimentos-fonte de cafeína e a prematuridade.

\section{Metodologia}

O presente estudo foi realizado na maternidade do Instituto Fernandes Figueira (IFF) na Cidade do Rio de Janeiro, Brasil, no período de julho de 2000 a abril de 2001. O IFF é a unidade materno-infantil da Fundação Oswaldo Cruz (FIOCRUZ) sendo referência para o tratamento de diversas doenças de alta complexidade. No ano de 2000 ocorreram 1.121 nascimentos (1,13\% do total de partos ocorridos no Município do Rio de Janeiro), destes $42,0 \%$ nasceram com peso inferior a $2.500 \mathrm{~g}$. A proporção de prematuridade dentre os de baixo peso foi de $89,3 \%$. $\mathrm{Na}$ distribuição da renda familiar das parturientes em 1999, 43,0\% apresentavam renda de até dois salários-mínimos e 38,0\% renda superior a cinco salários-mínimos.

Calculou-se que seriam necessárias $288 \mathrm{mu}$ lheres ( 144 casos e controles) para um erro alfa de $5,0 \%$, erro beta de $20,0 \%$, uma exposição nos controles de $40,0 \%$ e uma OR de 2,0 (valor esperado para alta exposição à cafeína 22,25,26,27) . Estimou-se um alto consumo de café no Rio de Janeiro, pois pesquisa com amostragem probabilística para o município mostrou que a média de consumo em adultos era de duas xícaras/dia com um desvio padrão de 2,3 , sendo que $90,0 \%$ dos participantes referiram consumo diário de café 28 . Adicionalmente, a média de cafeína em cafés brasileiros é de $37 \mathrm{mg}$ /xícara, com um desvio-padrão de 6,0 29.

O grupo de casos foi composto pelos recémnascidos vivos com idade gestacional (IG) inferior a 37 semanas de gestação (prematuros), nascidos na maternidade do IFF, no período do estudo, em qualquer dia da semana e horário. Os recém-nascidos portadores de síndromes ou malformações (genéticas, neurológicas, gastrointestinais, renais, polimalformados), crianças portadoras de infecções congênitas (toxoplasmose, rubéola, citomegalia, herpes), gêmeos, trigêmeos e crianças aidéticas foram excluídas do estudo. Os controles foram as crianças nascidas com 37 semanas ou mais de gestação (a termo), no mesmo período dos casos, durante o horário de rotina do hospital (7 às 16 horas), em três dias da semana. Esses horários foram escolhidos por atenderem 90,0\% das crianças nascidas a termo. Por questões logísticas, não foram incluídas crianças controles fora deste horário. Foram aplicados aos controles os mesmos critérios de exclusão dos casos.

As entrevistadoras (uma nutricionista e duas graduandas de nutrição), foram treinadas em relação ao preenchimento do questionário de freqüência de consumo alimentar (QFCA) e coleta das informações dos prontuários materno e neonatal. As entrevistadoras visitavam diariamente (inclusive nos finais de semana) a sala de pré-parto e, através do livro de registro de partos, identificavam e registravam os nascimentos ocorridos nas últimas 24 horas. As en- 
trevistas foram realizadas nas primeiras 72 horas de puerpério, antes da alta hospitalar. As informações dos prontuários materno (hipertensão e diabetes durante a gravidez) e neonatal (data e hora de nascimento do recém-nascido, data da última menstruação - DUM -, IG pela DUM, pelo método New Ballard - NB - e pela ultra-sonografia - USG -, classificação para a IG, sexo, tipo de nascimento e antropometria do recém-nascido) também foram colhidas durante este período.

Neste estudo, utilizou-se três instrumentos para estimar a IG: a DUM, conhecida com certeza pela mãe, ou o resultado da USG mais antiga realizada até a 20a semana de gestação ou o escore NB 30 . O primeiro critério para o cálculo da IG foi à utilização da DUM. Na falta desta informação ou a não certeza pela mãe, utilizava-se o resultado da USG e, por fim, o resultado do NB.

Para a coleta da informação sobre o consumo de cafeína na gravidez, foi utilizado um QFCA, semi-quantitativo, com porções padronizadas. O questionário foi previamente validado ${ }^{31}$ para aplicação em adultos, mas não em gestantes. Para sua utilização em gestantes realizou-se um estudo de calibração em 103 mulheres grávidas, atendidas na maternidade onde foi desenvolvido posteriormente o estudo. Segundo Willett \& Lenart 32 , uma alternativa para a validação do questionário é sua calibração usando um método aberto, como por exemplo, um único recordatório de 24 horas, como realizado na presente pesquisa. Dessa análise, 15 alimentos foram excluídos (milho verde, pipoca, camarão, limão, pão doce, polenta/angu, lentilha/ervilha/grão-de-bico, carne de porco, carne de boi com osso/mocotó/rabo, peixe enlatado, bacon/toucinho, maracujá, cebola, alho e pimentão), três foram incluídos (bananada/goiabada, adoçante e refrigerante light/diet), dois foram agrupados (doces, que passou a agrupar caramelo, chocolate em barra, bombom, pudim ou doce de leite, e bebidas alcoólicas, que passou a agrupar vinho, cerveja ou outras bebidas alcoólicas) e um foi desdobrado (queijo que foi desdobrado em minas/ricota e queijo prato/mussarela). O questionário inicial, com 80 itens ficou composto, para aplicação nas gestantes, de 64 itens, contudo, para o consumo de alimentos-fonte de cafeína o questionário não foi modificado.

Para o cálculo do consumo total de cafeína, considerou-se os seguintes alimentos e as suas respectivas padronizações: café (xícara de $50 \mathrm{ml}$ ), chá mate (copo pequeno de $165 \mathrm{ml}$ ) e chocolate em pó (colher de sobremesa com 11g). Para cada alimento, foram perguntadas a sua fre- qüência de consumo e a quantidade usualmente consumida durante toda a gravidez. Há evidências de que a média de consumo dos alimentos contendo cafeína permanece constante durante a gravidez $33,34,35$.

Para o cálculo da quantidade diária total de cafeína ingerida, considerou-se que: $50 \mathrm{ml}$ de café $=35,6 \mathrm{mg}$ de cafeína; $165 \mathrm{ml}$ de mate $=47,1 \mathrm{mg}$ de cafeína e $11 \mathrm{~g}$ de chocolate em pó $=6,5 \mathrm{mg}$ de cafeína 36.

Além da quantidade total diária de cafeína ingerida, também foram analisadas, individualmente, a freqüência de consumo diária de cada alimento-fonte de cafeína assim como a sua quantidade diária ingerida.

Informações sobre os fatores sócio-econômicos e demográficos, de saúde materna e de hábitos de vida durante a gestação foram obtidas através de entrevista no pós-parto. Idade materna foi categorizada em: inferior a 18, de 18 a 34 e superior a 34 anos, pois as categorias inferior a 18 e superior a 34 anos são consideradas como indicadoras de risco para prematuridade 37,38,39. A escolaridade materna foi avaliada em: sem escolaridade, 1a à $4 \underline{a}$ série, $5 \underline{a}$ à $8 \underline{a}$ série, 20 grau incompleto, 2 o grau completo e universitário e re-categorizada, devido ao pequeno número de mulheres nas categorias extremas, para $\leq 1$ o grau e > lo grau. O mesmo foi feito para renda. A raça foi avaliada pelo entrevistador com opções para: branca, parda e negra. Hipertensão arterial materna foi retirada do prontuário materno e tabagismo durante a entrevista. As mães que fumaram um ou mais dias foram consideradas fumantes. Avaliou-se tabagismo por trimestre e o número de cigarros fumados por dia.

Para comparar a prevalência de prematuridade entre as categorias de cada fator de risco a medida de efeito adotada foi a OR e IC95\%. A diferença entre as médias foi feita usando o teste $t$ de Student Os bancos de dados foram construídos no programa estatístico Epi Info versão 6.04 e analisados no programa estatístico SAS para Windows versão 8 .

O projeto de pesquisa foi submetido e aprovado pela Comissão de Ética em Pesquisa do IFF. Houve o consentimento livre e esclarecido das mães.

\section{Resultados}

A duração da gestação foi calculada em 85,4\% das mulheres pela DUM, 4,0\% pela USG e 10,6\% pelo método NB. A média da IG entre os casos foi de aproximadamente 33 semanas de gestação e entre os controles de 39 semanas. Entre todos os partos prematuros, $13,9 \%$ ocorreram 
com menos de 32 semanas de gestação e $32,4 \%$ ocorreram entre 32 e 36 semanas de gestação. Do total de mulheres selecionadas para o estudo, 12 mulheres $(3,9 \%)$ se recusaram a responder o QFCA (11 mães de crianças a termo e uma mãe de criança prematura). O consumo de álcool, referido por $7,6 \%$ das mulheres e o consumo de chocolate em barra, referido por somente $1,03 \%$ das mulheres não se associaram com prematuridade e não foram incluídos nas demais etapas do estudo. Diabetes durante a gravidez foi excluída da análise, pois não associou-se a prematuridade e somente $3,0 \%$ das mulheres a referiram.

Dos fatores estudados, somente a hipertensão arterial materna associou-se à prematuridade (OR = 2,58; IC95\%: 1,55-4,29) (Tabela 1).

Dado que alguns estudos sugerem que o efeito sobre a prematuridade pode ocorrer somente para partos muito prematuros, realizouse uma análise secundária quando estudou-se os mesmos fatores para partos que ocorreram antes da 32a semana de gestação. Também nessa análise, somente a hipertensão arterial materna associou-se ao parto antes da 32a semana de gestação (OR = 4,56; IC95\%: 2,10-9,93) e entre a 32 a e a 36 a semanas (OR $=2,0$; IC95\%: 1,10-3,65) (dados não apresentados).

Com relação ao consumo de cafeína, a média de consumo diário entre os casos foi de $56,2 \mathrm{mg}$ e entre os controles de 55,0mg ( $\mathrm{p}=0,89$ ). $\mathrm{O}$ alimento-fonte de cafeína mais consumido e o que mais contribuiu para a quantidade diária total ingerida de cafeína tanto entre os casos quanto entre os controles foi o café (Tabela 2). O chocolate em pó foi o segundo alimento-fonte de cafeína mais consumido, mas o mate foi o segundo alimento-fonte de cafeína que mais contribuiu para a quantidade diária total ingerida de cafeína, o que pode ser explicado pela maior quantidade de cafeína contida no mate.

A distribuição entre casos e controles para consumo total de cafeína, freqüência e quantidade por alimento-fonte de cafeína não foi estatisticamente diferente, exceção somente para o consumo de até um xícara de café/dia que associou-se a prematuridade, como fator de proteção (OR = 0,50; IC95\%: 0,27-0,92). Essa associação, sem significado biológico, deve-se, provavelmente, ao acaso (Tabela 3 ).

Observou-se uma associação entre tabagismo e consumo de cafeína diferente do relatado na literatura, particularmente entre os casos, onde quanto maior o número de cigarros fumados/dia, menor foi o consumo de cafeína/dia. Já entre os controles, aquelas que fumaram até nove cigarros/dia foram as que apresentaram maior consumo de cafeína/dia (Tabela 4).

\section{Discussão e conclusão}

Em consonância com a maioria dos estudos encontrados na literatura $34,40,41,42,43$, nossos achados não mostraram risco aumentado de prematuridade com consumo de cafeína.

Os nossos achados também não mostraram associação entre fatores sócio-econômicos e demográficos com a prematuridade, resultados consistentes com os achados de outros investigadores 44,45,46,47,48. Em artigo de revisão sobre os fatores associados a prematuridade, Berkowitz \& Papiernik 37 notaram ausência de associação ou achados inconsistentes para muitos dos fatores por nós estudados como idade materna, escolaridade, situação conjugal. O único fator que encontramos consistentemente associado com prematuridade foi a hipertensão arterial materna, associação referida em muitos estudos 46,49,50,51.

Possíveis limitações do estudo incluem: (1) não colhemos informação de outros alimentos-fonte de cafeína, como refrigerante do tipo cola e chá preto e nem de medicação contendo cafeína. Entretanto, Camargo et al. 52, em um dos poucos estudos sobre o consumo de cafeína em alimentos nacionais, em uma amostra representativa com seiscentos indivíduos entre 9 e 80 anos, mostraram que, para todas as faixas etárias, o café foi a principal fonte de cafeína, o que pode ser atribuído aos maiores níveis de cafeína no café quando comparado a outras fontes. Apesar disso, informação detalhada do método de preparação e marca dos alimentosfonte de cafeína seriam úteis para uma melhor quantificação da exposição à cafeína; (2) não foram também medidos os níveis corporais de cafeína. Entretanto, Little et al. 53, estudando a concordância entre os auto-relatos do uso de cafeína e níveis urinários de cafeína em mulheres no puerpério, encontraram que enquanto a obtenção de um teste de urina positivo para não-consumidoras de cafeína foi improvável, um teste de urina negativo foi comum para usuárias de cafeína; (3) a informação foi coletada retrospectivamente, não se podendo excluir a possibilidade de viés de memória, tanto com relação à DUM, quanto em relação ao consumo dos alimentos-fonte de cafeína. Um viés de memória seria mais provável caso ocorresse alteração de consumo durante a gestação, contudo, há evidências na literatura de que a média de consumo dos alimentos contendo cafeína permanece constante durante a gravidez $33,34,35$. Adicionalmente, é pouco provável que tenha ocorrido viés diferencial entre casos e controles, uma vez que os alimentos faziam parte de uma longa lista de alimentos sem nenhuma conotação deletéria. 
Tabela 1

Distribuição entre casos e controles e odds ratio (OR) para fatores sócio-econômicos e demográficos, hipertensão arterial materna e tabagismo em mulheres atendidas em um hospital público do Rio de Janeiro, Brasil, 2000/2001.

\begin{tabular}{|c|c|c|c|c|c|}
\hline \multirow[t]{2}{*}{ Variável } & \multicolumn{2}{|c|}{ Casos } & \multicolumn{2}{|c|}{ Controles } & \multirow[t]{2}{*}{ OR (IC95\%) } \\
\hline & $n$ & $\%$ & $\mathrm{n}$ & $\%$ & \\
\hline \multicolumn{6}{|l|}{ Idade materna (anos) } \\
\hline$<18$ & 15 & 10,8 & 30 & 18,6 & $0,52(0,25-1,07)$ \\
\hline $18-34$ & 92 & 66,2 & 95 & 58,6 & 1,00 \\
\hline$>34$ & 32 & 23,0 & 37 & 22,8 & $0,89(0,49-1,61)$ \\
\hline \multicolumn{6}{|l|}{ Escolaridade materna } \\
\hline$>1$ o grau & 65 & 47,8 & 80 & 53,0 & 1,00 \\
\hline$\leq 1$ o grau & 71 & 52,2 & 71 & 47,0 & $1,23(0,77-1,95)$ \\
\hline \multicolumn{6}{|l|}{ Cor da pele } \\
\hline Branca & 58 & 43,3 & 75 & 48,4 & 1,00 \\
\hline Parda & 56 & 41,8 & 47 & 30,3 & $1,54(0,89-2,67)$ \\
\hline Negra & 20 & 14,9 & 33 & 21,3 & $0,78(0,39-1,58)$ \\
\hline \multicolumn{6}{|c|}{ Renda per capita (salários mínimos) } \\
\hline$\geq 1$ & 78 & 67,2 & 77 & 61,1 & 1,00 \\
\hline$<1$ & 38 & 32,8 & 49 & 38,9 & $0,76(0,45-1,29)$ \\
\hline \multicolumn{6}{|l|}{ Situação conjugal } \\
\hline Com companheiro & 114 & 84,4 & 118 & 78,2 & 1,00 \\
\hline Sem companheiro & 21 & 15,6 & 33 & 21,8 & $0,65(0,35-1,20)$ \\
\hline \multicolumn{6}{|l|}{ Hipertensão } \\
\hline Não & 83 & 59,3 & 128 & 79,0 & 1,00 \\
\hline $\operatorname{Sim}$ & 57 & 40,7 & 34 & 21,0 & $2,58(1,55-4,29)$ \\
\hline \multicolumn{6}{|l|}{ Tabagismo } \\
\hline Não & 111 & 79,9 & 140 & 86,4 & 1,00 \\
\hline Sim & 28 & 20,1 & 22 & 13,6 & $1,60(0,87-2,95)$ \\
\hline
\end{tabular}

Tabela 2

Proporção e média diária da quantidade de cafeína ingerida por alimento-fonte de cafeína entre casos e controles em mulheres atendidas em um hospital público do Rio de Janeiro, Brasil, 2000/2001.

\begin{tabular}{|c|c|c|c|c|c|c|}
\hline \multirow[t]{2}{*}{ Alimento-fonte } & \multicolumn{3}{|c|}{ Casos } & \multicolumn{3}{|c|}{ Controles } \\
\hline & $n$ & $\%$ & $\mathrm{mg} / \mathrm{dia}$ & $\mathrm{n}$ & $\%$ & $\mathrm{mg} / \mathrm{dia}$ \\
\hline Café & $88 / 134$ & 65,7 & 40,0 & $113 / 156$ & 72,4 & 41,0 \\
\hline Mate & $25 / 134$ & 18,7 & 11,2 & $34 / 156$ & 21,8 & 9,8 \\
\hline Chocolate em pó & $66 / 134$ & 49,3 & 4,9 & $63 / 156$ & 46,8 & 4,2 \\
\hline
\end{tabular}


Distribuição entre casos e controles e odds ratio (OR) para consumo diário total de cafeína e de alimentos-fonte de cafeína em mulheres atendidas em um hospital público do Rio de Janeiro, Brasil, 2000/2001.

\begin{tabular}{|c|c|c|c|c|c|}
\hline \multirow[t]{2}{*}{ Variável } & \multicolumn{2}{|c|}{ Casos } & \multicolumn{2}{|c|}{ Controles } & \multirow[t]{2}{*}{ OR (IC95\%) } \\
\hline & $\mathrm{n}$ & $\%$ & $\mathrm{n}$ & $\%$ & \\
\hline \multicolumn{6}{|c|}{ Quantidade diária total de cafeína (mg) } \\
\hline Até 50 & 74 & 55,2 & 102 & 65,4 & 1,00 \\
\hline $50-99$ & 39 & 29,1 & 34 & 21,8 & $1,58(0,88-2,84)$ \\
\hline$\geq 100$ & 21 & 15,7 & 20 & 12,8 & $1,35(0,48-3,80)$ \\
\hline \multicolumn{6}{|c|}{ Freqüência diária de café } \\
\hline Nenhuma & 46 & 34,3 & 43 & 27,6 & 1,00 \\
\hline Até 1 vez & 48 & 35,8 & 79 & 50,6 & $0,57(0,32-1,02)$ \\
\hline 2 vezes ou mais & 40 & 29,9 & 34 & 21,8 & $1,10(0,57-2,14)$ \\
\hline \multicolumn{6}{|l|}{ Xícaras de café/dia } \\
\hline Nenhuma & 46 & 34,3 & 43 & 27,5 & 1,00 \\
\hline Até 1 xícara & 39 & 29,1 & 73 & 46,8 & $0,50(0,27-0,92)$ \\
\hline 1 a 2 xícaras & 7 & 5,2 & 4 & 2,6 & $1,64(0,39-7,24)$ \\
\hline 3 xícaras ou mais & 42 & 31,4 & 36 & 23,1 & $1,09(0,57-2,10)$ \\
\hline \multicolumn{6}{|c|}{ Freqüência diária de mate } \\
\hline Nenhuma & 109 & 81,4 & 122 & 78,2 & 1,00 \\
\hline Até 1 vez & 20 & 14,9 & 27 & 17,3 & $0,83(0,42-1,63)$ \\
\hline 2 vezes ou mais & 5 & 3,7 & 7 & 4,5 & $0,80(0,21-2,91)$ \\
\hline \multicolumn{6}{|l|}{ Copos de mate/dia } \\
\hline Nenhum & 109 & 81,3 & 123 & 78,9 & 1,00 \\
\hline Até 1 copo & 16 & 11,9 & 25 & 16,0 & $0,72(0,35-1,49)$ \\
\hline 2 copos ou mais & 9 & 6,7 & 8 & 5,1 & $1,27(0,43-3,77)$ \\
\hline \multicolumn{6}{|c|}{ Freqüência diária de chocolate em pó } \\
\hline Nenhuma & 68 & 50,7 & 83 & 53,2 & 1,00 \\
\hline Até 1 vez & 53 & 39,6 & 63 & 40,4 & $1,02(0,58-1,79)$ \\
\hline 2 vezes ou mais & 13 & 9,7 & 10 & 6,4 & $1,48(0,73-2,98)$ \\
\hline \multicolumn{6}{|c|}{ Colheres de chocolate em pó/dia } \\
\hline Nenhuma & 68 & 50,7 & 85 & 54,5 & 1,00 \\
\hline Até 1 colher & 38 & 28,4 & 41 & 26,3 & $1,16(0,65-2,07)$ \\
\hline Até 2 colheres & 28 & 20,9 & 30 & 19,2 & $1,17(0,61-2,23)$ \\
\hline
\end{tabular}

Tabela 4

Média da quantidade diária total de cafeína ingerida segundo o número de cigarros fumados por dia entre casos e controles em mulheres atendidas em um hospital público do Rio de Janeiro, Brasil, 2000/2001.

\begin{tabular}{|c|c|c|c|c|c|c|}
\hline \multirow[t]{2}{*}{ Cigarros/dia } & \multicolumn{3}{|c|}{ Casos } & \multicolumn{3}{|c|}{ Controles } \\
\hline & $\mathrm{n}$ & $\%$ & mg/dia & $n$ & $\%$ & $\mathrm{mg} / \mathrm{dia}$ \\
\hline 0 & 107 & 80,4 & 58,5 & 135 & 86,5 & 55,3 \\
\hline 0,1 a 9 & 15 & 11,3 & 56,2 & 15 & 9,6 & 57,6 \\
\hline$\geq 10$ & 11 & 8,3 & 34,9 & 6 & 3,9 & 41,7 \\
\hline
\end{tabular}


O café contém muitos componentes químicos diferentes, sendo a cafeína o principal princípio ativo. Consideramos a quantidade de cafeína total assim como a freqüência e quantidade de cada alimento-fonte de cafeína separadamente e não encontramos nenhuma associação estatisticamente significante. Brown et al. 54 mostraram que medir o consumo de café ao invés do consumo da cafeína, por exemplo, pode contribuir para a falta de achados positivos nos estudos de café como um fator de risco quando a cafeína é a exposição de interesse. Fortier et al. 34 encontraram que o café descafeinado não foi associado com restrição de crescimento intrauterino, ao contrário do café cafeinado. Eskenazi et al. 27 mostraram que o consumo de café cafeinado foi associado com diminuição da gestação e do peso ao nascer, e que o uso de café descafeinado durante a gravidez não mostrou associação com esses dois desenlaces. A utilização de café descafeinado nessa população se ocorrer, é mínima.

No presente estudo, as mulheres que menos fumaram foram as que mais consumiram cafeína. Esse achado pode decorrer do pequeno número de mães fumantes ou de um viés de informação. Morrison 55 considera que, embora o tabagismo seja percebido como socialmente não-desejável, o consumo de cafeína não o é. Adicionalmente, ao contrário do que ocorre nos Estados Unidos, no Brasil não há nenhuma restrição ao consumo de cafeína durante a gestação pelos órgãos competentes. Por isso, o consumo de cafeína pode ser melhor informado e, talvez, as mulheres tenham sub-relatado o seu tabagismo. Ford et al. 56 e Foss et al. 57, estudando a confiabilidade do relato de tabagismo na gravidez através de marcadores bioquímicos, encontraram que as grávidas fumantes muitas vezes negam o tabagismo durante a gravidez e quando o assumem, o fazem de modo subestimado. Kesmodel \& Olsen 58 encontraram que a acurácia das informações sobre tabagismo diminuiu particularmente entre as que mais fumaram durante a gravidez.

Segundo alguns autores 59,60,61, estudos sobre prematuridade deveriam subdividi-la em prematuridade resultante da ruptura precoce das membranas ovulares (RPMO), prematuridade espontânea e prematuridade resultante de intervenção médica. Klebanoff \& Shiono 62 apontaram, entretanto, que a apresentação clínica pode refletir o tempo de diagnóstico e acesso à saúde mais do que diferenças etiológicas, e que uma maior prevalência de prematuridade após RPMO entre mulheres negras, por exemplo, pode ser devido a pior atenção médica. Essas autoras também sugeriram que pode haver coincidência etiológica entre prematuridade espontânea e prematuridade induzida por questões médicas, isto é, uma proporção de partos indicados por questões médicas poderia ter sido um parto prematuro espontâneo na ausência de intervenção médica, assim como alguns partos prematuros espontâneos teriam sido medicamente induzidos se essas mulheres tivessem se apresentado precocemente à maternidade. Além disso, alguns fatores de risco para parto induzido (ex.: hipertensão) também são fatores de risco para prematuridade espontânea. Nas análises secundárias realizadas onde somente partos muito prematuros foram analisados, também não houve associação com o consumo de cafeína, o que indica que a ausência de associação não decorre de erro de classificação para a prematuridade.

A maioria dos estudos 23,24,43,63,64 sugere que o consumo de cafeína a níveis moderados pelas mulheres grávidas não afeta adversamente o feto. Entretanto, doses de 200mg de cafeína significantemente reduziram o fluxo sangüíneo na placenta, provavelmente através de vasoconstricção o que pode diminuir o crescimento fetal 13. É possível ainda que as diferenças encontradas entre os estudos sejam devidas, em parte, às diferenças entre as populações no preparo de café. Camargo \& Toledo 29, avaliando a quantidade de cafeína nos diferentes tipos de café normalmente consumidos pela população brasileira, observaram que houve uma grande variabilidade existente com relação ao teor deste alcalóide, devido à quantidade de pó utilizada, ao tipo de café e a forma de preparo do mesmo. Bracken et al. 65 observaram variação até quando o mesmo respondente fervia o café ou chá em condições aparentemente idênticas no mesmo dia.

Embora o consumo de cafeína seja bastante difundido, com a maioria das mulheres avaliadas tendo consumido cafeína durante a gravidez, o nível de consumo foi baixo. Doses maiores do que $100 \mathrm{mg}$ /dia foram reportadas por menos de $15,0 \%$ das mulheres estudadas e tudo indica que o efeito deletério da cafeína ocorreria somente em grandes doses 22,25,63, o que não foi possível testar na presente análise.

Doses acima de $300 \mathrm{mg} /$ dia têm sido mais consistentemente associadas à prematuridade, mas no Brasil, onde o consumo de cafeína é freqüente, a quantidade de consumo nessa faixa é extremamente baixa. Dado o padrão de consumo observado no presente estudo, difundir o não-consumo de cafeína em gestantes brasileiras não se justifica. 


\section{Resumo}

A cafeína (1,3,7-trimetilxantina) é um alcalóide que facilmente atravessa a barreira placentária podendo interferir no crescimento e desenvolvimento das células fetais e comprometer a oxigenação fetal. Considerando o amplo consumo de alimentos que contêm cafeína no Brasil, o objetivo do estudo foi avaliar a associação entre o consumo total de cafeína e de alimentos-fonte de cafeína com a prematuridade. Um estudo caso-controle de 140 casos (recém-nascidos com idade gestacional inferior a 37 semanas de gestação) e 162 controles (recém-nascidos com 37 semanas ou mais) avaliou o consumo de cafeína durante a gravidez. $\mathrm{Pa}$ ra se medir o consumo utilizou-se um questionário de freqüência alimentar, semi-quantitativo, baseado nos seguintes alimentos: café, chá mate e chocolate em pó. O consumo total de cafeina e de alimentos-fonte de cafeína durante a gravidez não foram associados à prematuridade, com a maioria das mulheres tendo consumido menos que $300 \mathrm{mg} /$ dia. O consumo de cafeína observado no presente estudo não suporta recomendações contra o consumo de cafeína em gestantes brasileiras.

Cafeína; Gravidez; Trabalho de Parto Prematuro

\section{Colaboradores}

As autoras participaram da concepção do projeto de pesquisa, assim como na análise e interpretação dos dados, além da revisão de texto. R. A. G. Souza participou, também, do trabalho de campo no Instituto Fernandes Figueira.

\section{Agradecimentos}

Ao Conselho Nacional de Desenvolvimento Científico e Tecnológico (CNPq) pelo apoio financeiro e ao Instituto Fernandes Figueira.

\section{Referências}

1. Heller J. What do we know about the risks of caffeine consumption in pregnancy? Br J Addict 1987; 82:885-9.

2. Dlugosz L, Bracken MB. Reproductive effects of caffeine: a review and theoretical analysis. Epidemiol Rev 1992; 14:83-100.

3. Goyan JE. Food and Drug Administration news release number P80-36. Washington DC: Food and Drug Administration; 1980.

4. Fernandes O, Sabharwal M, Smiley T, Pastuszak A, Koren G, Einarson T. Moderate to heavy caffeine consumption during pregnancy and relationship to spontaneous abortion and abnormal fetal growth: a meta-analysis. Reprod Toxicol 1998; 12:435-44.

5. Barone JJ, Roberts HR. Caffeine consumption. Food Chem Toxicol 1996; 34:119-29.

6. Srisuphan W. Caffeine consumption during pregnancy and association with miscarriage $[\mathrm{PhD}$ Thesis]. New Haven: Yale University; 1983.

7. James JE. Caffeine and health. In: James JE, editor. Human reproduction. London: Academic Press; 1991. p. 219-44.

8. Knutti R, Rothweiler H, Schlatter C. The effect of pregnancy on the pharmacokinetics of caffeine. Arch Toxicol Suppl 1982; 5:187-92.

9. Brazier JL, Ritter J, Berland M, Khenfer D, Faucon G. Pharmacokinetics of caffeine during and after pregnancy. Dev Pharmacol Ther 1983; 6:313-22.

10. Beaulac-Baillargeon L, Desrosiers C. Caffeine-cigarette interaction on fetal growth. Am J Obstet Gynecol 1987; 157:1236-40.

11. Snyder SH. Adenosine as a mediator of the behavioral effects of xanthines. In: Dews PB, editor. Caffeine. New York: Springer-Verlag; 1984. p. 12941.

12. Sutherland DJ, McPherson DD, Renton KW, Spencer CA, Montague TJ. The effect of caffeine on cardiac rate, rhythm, and ventricular repolarization: analysis of 18 normal subjects and 18 patients with primary ventricular dysrhythmia. Chest 1985; 87:319-24.

13. Kirkinen P, Jouppila P, Koivula A, Vuori J, Puukka $M$. The effect of caffeine on placental and fetal blood flow in human pregnancy. Am J Obstet Gynecol 1983; 147:939-42.

14. Kihlman BA, editor. Caffeine and chromosomes. Amsterdam: Elsevier Scientific Publishing Co.; 1977.

15. Dlugosz L, Belanger K, Hellenbrand, Holford TR, Leaderer B, Bracken MB. Maternal caffeine consumption and spontaneous abortion: a prospective cohort study. Epidemiol 1996; 7:250-5.

16. Christian MS, Brent RL. Teratogen update: evaluation of the reproductive and developmental risks of caffeine. Teratology 2001; 64:51-78.

17. Adler ID. The problem of caffeine mutagenicity. In: Vogel F, Rohrborn G, editors. Chemical mutagenesis in mammals and man. New York: SpringerVerlag; 1970. p. 353-403.

18. Schlegel R, Pardee AB. Caffeine-induced uncoupling of mitosis from the completion of DNA replication in mammalian cells. Science 1986; 232 : 1264-6. 
19. Adler ID, Wasson JS, Malling HV. Literature checklist on the genetic effect of caffeine. Environ $\mathrm{Mu}-$ tagen 1971; 4:44-50.

20. Kihlman BA. Second international caffeine workshop report on mutagenicity. Nutr Rev 1980; 38: 196-200.

21. Weathersbee PS, Olsen LK, Lodge JR. Caffeine and pregnancy: a retrospective survey. Postgrad Med 1977; 62:64-9.

22. Williams MA, Mittendorf R, Stubblefield PG, Lieberman E, Schoenbaum SC, Monson RR. Cigarettes, coffee, and preterm premature rupture of the membranes. Am J Epidemiol 1992, 135:895903.

23. Bracken MB, Triche EW, Belanger K, Hellenbrand $\mathrm{K}$, Leaderer BP. Association of maternal caffeine consumption with decrements in fetal growth. Am J Epidemiol 2003; 157:456-66.

24. Santos IS, Victora CG, Huttly S, Morris S. Caffeine intake and pregnancy outcomes: a meta-analytic review. Cad Saúde Pública 1998; 14:523-30.

25. Van Den Berg BJ. Epidemiologic observations of prematurity: effects of tabacco, coffee and alcohol. In: Reed MD, Stanley FJ, editors. The epidemiology of prematurity. Baltimore: Urban \& Schwarzenberg; 1977. p. 157-76.

26. Fenster L, Eskenazi B, Windham GC, Swan SH. Caffeine consumption during pregnancy and fetal growth. Am J Public Health 1991; 81:458-61.

27. Eskenazi B, Stapleton AL, Kharrazi M, Chee WY. Associations between maternal decaffeinated and caffeinated coffee consumption and fetal growth and gestational duration. Epidemiol 1999; 10:242-9.

28. Sichieri R. Dietary patterns and their associations with obesity in the Brazilian city of Rio de Janeiro. Obesity Research 2002; 10:42-8.

29. Camargo MCR, Toledo MCF. Teor de cafeína em cafés brasileiros. Cienc Tecnol Aliment 1998; 18:421-4.

30. Ballard JL, Khoury JC, Wedig K, Wang L, EilersWalsman BL, Lipp R. New Ballard score, expanded to include extremely premature infants. J Pediatr 1991; 119:417-23.

31. Sichieri R. Estudo de validação do questionário de freqüência de consumo de alimentos. In: Sichieri R, organizadora. Epidemiologia da obesidade. Rio de Janeiro: Eduerj; 1998. p. 25-34.

32. Willett W, Lenart E. Reproducibility and validity of food-frequency questionnaires. In: Willett W, editor. Nutritional epidemiology. New York: Oxford University Press; 1998. p. 101-47.

33. Watkinson B, Fried PA. Maternal caffeine use before, during and after pregnancy and effects upon offspring. Neurobehav Toxicol Teratol 1985; 7:9-17.

34. Fortier I, Marcoux S, Beaulac-Baillargeon L. Relation of caffeine intake during pregnancy to intrauterine growth retardation and preterm birth. Am J Epidemiol 1993; 137:931-40.

35. Erkkola M, Karppinen M, Javanainen J, Rasanen L, Knip M, Virtanen SM. Validity and reproducibility of a food frequency questionnaire for pregnant Finnish women. Am J Epidemiol 2001; 154:466-76.

36. Bunker ML, McWilliams M. Caffeine content of common beverages. J Am Diet Assoc 1979; 74:2832.

37. Berkowitz GS, Papiernik E. Epidemiology of preterm birth. Epidemiol Rev 1993; 15:414-43.

38. Pschirrer R, Monga M. Risk factors for preterm labor. Clin Obstet Gynecol 2000; 43:727-34.

39. Kilsztajn S, Rossbach A, Carmo MSN, Sugahara GTL. Assistência pré-natal, baixo peso e prematuridade no Estado de São Paulo, 2000. Rev Saúde Pública 2003; 37:303-10.

40. Berkowitz GS, Holford TR, Berkowitz RL. Effects of cigarette smoking, alcohol, coffee and tea consumption on preterm delivery. Hum Dev 1982; 7:239-50.

41. Peacock JL, Bland JM, Anderson HR. Preterm delivery: effects of socioeconomic factors, psychological stress, smoking, alcohol, and caffeine. BMJ 1995; 311:531-6.

42. Bicalho GG, Barros Filho AA. Peso ao nascer e influência do consumo de cafeína. Rev Saúde Pública 2002; 36:180-7.

43. Clausson B, Granath F, Ekbom A, Lundgreen S, Nordmark A, Signorello LB, et al. Effect of caffeine exposure during pregnancy on birth weight and gestational age. Am J Epidemiol 2002; 155:429-36.

44. Kramer MS. Determinants of low birth weight: methodological assessment and meta-analysis. Bull World Health Organ 1987; 65:663-737.

45. Wen SW, Goldenberg RL, Cutter GR, Hoffman HJ, Cliver SP. Intrauterine growth retardation and preterm delivery: prenatal risk factors in an indigent population. Am J Obstet Gynecol 1990; 162:213-8

46. Mavalankar DV, Gray RH, Trivedi CR. Risk factors for preterm and term low birth-weight in Ahmedabad, India. Int J Epidemiol 1992; 21:263-72.

47. Adams MM, Sarno AP, Harlass FE, Rawlingsa JS, Read JA. Risk factors for preterm delivery in a healthy cohort. Epidemiol 1995; 6:525-32.

48. Wildschut HIJ, Nas T, Golding J. Are sociodemographic factors predictive of preterm birth? A reappraisal of the 1958 British Perinatal Mortality Survey. Br J Obstet Gynaecol 1997; 104:57-63.

49. Hartikainen-Sorri AL, Sorri M. Occupational and socio-medical factors in preterm birth. Obstet Gynecol 1989; 74:13-6.

50. Kramer MS, McLean FH, Eason EL, Usher RH. Maternal nutrition and spontaneous preterm birth. Am J Epidemiol 1992; 136:574-83.

51. Ray JG, Burrows RF, Burrows EA, Vermeulen MJ. MOS HIP: McMaster outcome study of hypertension in pregnancy. Early Hum Dev 2001; 64:129-43.

52. Camargo MCR, Toledo MCF, Farah HG. Caffeine daily intake from dietary sources in Brazil. Food Addit Contam 1999; 16:79-87.

53. Little RE, Uhl CN, Labbe RF, Abkowitz JL, Phillips EL. Agreement between laboratory tests and selfreports of alcohol, tobacco, caffeine, marijuana and other drug use in post-partum women. Soc Sci Med 1986; 22:91-8.

54. Brown J, Kreiger N, Darlington GA, Sloon M. Misclassification of exposure: coffee as a surrogate for caffeine intake. Am J Epidemiol 2001; 153:81520. 
55. Morrison AS. Control of cigarette smoking in evaluating the association of coffee drinking and bladder cancer. In: MacMahon B, Suginura T, editors. Coffee and health. New York: Cold Spring Harbor Laboratory; 1984. p. 127-34.

56. Ford RP, Tappin DM, Schluter PJ, Wild CJ. Smoking during pregnancy: how reliable are maternal self reports in New Zealand? J Epidemiol Community Health 1997; 51:246-51.

57. Foss RP, Haug K, Hesla PE, Lund-Larsen PG, Vasli LR. Can we rely on self-reported smoking habits? Tidsskr Nor Laegeforen 1998; 118:2165-8.

58. Kesmodel U, Olsen SF. Smoking habits among pregnant Danish women: reliability of information recorded after delivery. J Epidemiol Community Health 1999; 53:239-42.

59. Berkowitz GS, Black-Prince C, Lapinski RH, Savitz DA. Risk factors for preterm birth subtypes. Epidemiol 1998; 9:279-85.

60. Pickett KE, Abrams B, Selvin S. Defining preterm delivery - the epidemiology of clinical presentation. Paediatr Perinatal Epidemiol 2000; 14:305-8.
61. Ferguson SE, Smith EM, Salenieks ME, Windrin R, Walker MC. Preterm premature rupture of membranes. Nutritional and socioeconomic factors. Obstet Gynecol 2002; 100:1250-6.

62. Klebanoff M, Shiono P. Top down, bottom up, and inside out: reflections on preterm birth. Paediatr Perinatal Epidemiol 1995; 9:125-9.

63. Linn S, Schoenbaum SC, Monson RR, Rosner B, Stubblefield PG, Ryan KJ. No association between coffee consumption and adverse outcomes of pregnancy. N Engl J Med 1982; 306:141-5.

64. Santos IS, Victora CG, Huttly S, Carvalhal JB. Caffeine intake and low birth weight: a populationbased case-control study. Am J Epidemiol 1998; 147:620-7.

65. Bracken MB, Triche EW, Grosso L, Hellenbrand K, Belanger K, Leaderer BP. Heterogeneity in assessing self-reports of caffeine exposure: implications for studies of health effects. Epidemiol 2002; 13: 165-71.

Recebido em 16/Jun/2004

Versão final reapresentada em 20/Abr/2005

Aprovado em 16/Mai/2005 